\title{
Revisión
}

\section{Living Senior Labs, ecosistemas de co-creación e innovación abierta con personas mayores: revisión sistemática de la literatura en Ciencias Sociales}

Living Senior Labs, co-criação e ecossistemas de inovação aberta com idosos: revisão sistemática da literatura em Ciências Sociais (resumo: p. 19) Living Senior Labs, ecosystems of co-creation and open innovation with older people: a systematic review on Social Science literature (abstract: p. 19)

Antonio Víctor Martín-García(a)

<avmg@usal.es>

Bárbara Mariana Gutiérrez-Pérez ${ }^{(\text {b) }}$

<barbaragutierrez@usal.es> (iD)

Juan Carlos Aceros ${ }^{(\mathrm{c})}$

<jacerosg@uis.edu.co> (a,b) Departamento de Teoría e Historia de la Educación, Facultad de Educación, Universidad de Salamanca. Paseo de Canalejas, 169. Salamanca, España. 37008.

(c) Escuela de Trabajo Social, Universidad Industrial de Santander. Santander, Colombia.

Los Living Labs son experiencias colaborativas que buscan implicar a la ciudadanía en la gobernanza científica y la evaluación de tecnologías. A pesar de su interés, se sabe muy poco sobre estas comunidades, su funcionamiento, tipología y características. Por ello, se realizó una revisión sistemática de la literatura sobre de un tipo particular de Living Lab, orientado hacia las personas mayores: los Living Senior Labs. A partir de una búsqueda general en las principales bases de datos científicas (WOS y Scopus), y de la aplicación de criterios de inclusión preestablecidos tras la primera selección quedaron finalmente seleccionados 19 estudios sobre Senior Labs (2010 y 2021). Los resultados proporcionan un mejor conocimiento de este tipo de ecosistemas y crean una base firme para avanzar en el conocimiento de este campo.

Palabras clave: Living labs. Senior labs. Personas mayores. Innovación abierta. Co-creación. 


\section{Introducción}

Con el nombre de "Living Lab" se conoce uno de los actuales protagonistas de la innovación abierta. Algunos autores se refieren a dicho protagonista como un entorno, mientras que otros hablan de una metodología, un enfoque de innovación, una organización, o una red o sistema de innovación ${ }^{1-4}$. Independientemente de la definición concreta, la palabra "Living Lab" remite a la participación de diversos actores en proyectos de diseño de nuevos productos y servicios, con un enfoque centrado en el usuario 5 . Tal cosa implica contar con entornos muy parecidos a aquellos en los que se desenvuelve la vida cotidiana de la gente, así como el establecimiento de ecosistemas de co-creación, más o menos amplios, en los que se involucran las empresas, las universidades, los gobiernos y los ciudadanos o potenciales usuarios.

La filosofía y las prácticas de innovación abierta asociadas a los Living Lab han tenido una buena acogida en la última década en diversos países. En el contexto europeo, en el año 2006 se fundó la European Network of Living Labs (ENoLL), convirtiéndose en la federación internacional más relevante que promueve este modelo de innovación abierta ${ }^{6}$. Para ENoLL los Living Labs son entendidos como ecosistemas de co-creación destinados a la innovación y el desarrollo de experimentos. El objetivo principal de estos entornos es recopilar información y datos que se caracterizan por ser más precisos y ecológicamente válidos, al ser obtenidos mediante la observación de las diferentes actividades realizadas por los usuarios en condiciones muy similares a las reales y naturales ${ }^{5,7}$.

\section{¿Qué es un Living Lab?}

Como se ha mencionado previamente, existen diversas formas de entender lo que es un Living Lab. La primera conceptualización al respecto fue propuesta por William Mitchell, profesor del Instituto de Tecnología de Massachusetts (MIT), definiéndolo como una metodología de investigación centrada en el usuario y utilizada para detectar, validar, probar y refinar soluciones complejas dentro de contextos de vida real ${ }^{8}$. Desde la visión de los Living Labs como metodologías, Ståhlbröst ${ }^{9}$ los define como un enfoque de investigación específico que apoya la participación del usuario y los procesos de innovación desarrollados en contextos del mundo real. En estos procesos se cuenta con la implicación de cuatro tipos de partes interesadas: investigadores, empresas, usuarios y organizaciones públicas.

Más recientemente, sin embargo, se ha popularizado una comprensión de los Living Labs no como metodologías, sino como entornos, físicos o virtuales, en los que la metodología de la innovación abierta es implementada. Así los entienden, por ejemplo, Leminen y Westerlund ${ }^{3}$, para quienes los Living Labs son escenarios en los que las partes interesadas forman asociaciones y colaboraciones para la co-creación de prototipos, validación y prueba de nuevas tecnologías, servicios, productos o sistemas, todo ello en contextos de vida cotidiana. Por su parte, Bergvall-Kåreborn et al. ${ }^{1}$ hacen referencia a los Living Labs como entornos de innovación, centrados en el usuario y basados en la práctica e investigación continua, mediante un enfoque que facilita la influencia del usuario en los procesos de innovación abierta y con el objetivo de crear productos y servicios sostenibles. 
En estas definiciones, podemos encontrar tres denominadores comunes: la importancia que le dan al proceso de innovación abierta, la relevancia que le atribuyen al empleo de entornos o contextos reales de experimentación y su apuesta por la participación del usuario y destinatario final de los productos y/o desarrollos. Así, en primer lugar, mediante la innovación abierta los Living Labs responden a una insistente demanda por democratizar la tecnociencia, implicando a la ciudadanía en la gobernanza científica y la evaluación de tecnologías ${ }^{10}$. En este sentido, se enmarcan en preocupaciones abordadas extensamente por los estudios sociales de la ciencia y la tecnología (CTS o STS en inglés) y de gestión de la innovación. Los CTS constituyen un campo de estudio interesado por las mutuas relaciones entre los fenómenos sociales, los procesos de generación de conocimiento científico y las dinámicas de producción tecnológica.

En segundo lugar, los entornos reales de experimentación son considerados como un componente clave en los Living Labs ${ }^{11}$. La experimentación desarrollada en estos enclaves proporciona a los investigadores información detallada sobre una amplia gama de aspectos y elementos contextuales que pueden influir en la experiencia de los usuarios ${ }^{4,12}$. En consecuencia, el entorno se analiza o determina no como un elemento de información estático, sino como un elemento relacional que surge de una actividad (interacción entre el usuario y el producto o servicio), compuesto por diferentes dimensiones como: contexto temporal, contexto físico, contexto técnico o de información, contexto social y contexto de la actividad ${ }^{11}$.

En tercer lugar, otra característica propia de los entornos Living Labs corresponde a la participación explícita, necesaria y fundamental de los usuarios y destinatarios finales de los productos diseñados y testados, así como la participación de diferentes profesionales. Todo ello trata de favorecer el intercambio de conocimientos, así como el diseño de productos y servicios eficaces que faciliten la vida de las personas. En suma, el objetivo es resolver problemas específicos de los usuarios de una forma coherente para la mejora de su calidad de vida y bienestar ${ }^{1-4,6,7,9,13}$.

\section{Componentes básicos del entorno del Living Lab}

Partiendo de algunos de los rasgos previamente expuestos, Følstad ${ }^{12}$ y Veeckman et al. ${ }^{4}$ establecen lo que denominan The Living Lab Triangle. Este marco propone los siguientes tres pilares que, según los autores, deben conformar la base del diseño de todo Living Lab. Dichos pilares son: (1) los componentes básicos del entorno; (2) los componentes básicos del enfoque y (3) el resultado de la innovación. (ver, Figura 1) 


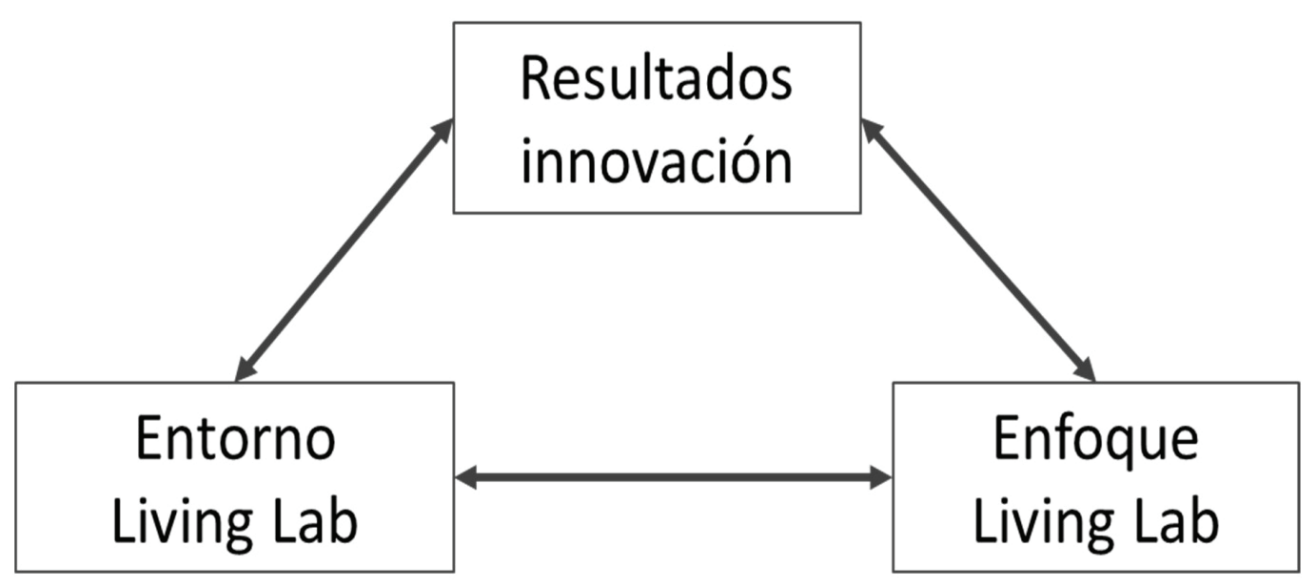

Figura 1. The living lab triangle

Fuente: Veeckman et al.4

En primer lugar, los componentes básicos del Living Lab se refieren a sus elementos materiales, inmateriales y contextuales ${ }^{4}$. Tal cosa incluye:

- Infraestructura técnica: Condiciones materiales básicas que deben estar a la disposición de todos los usuarios dentro del entorno del laboratorio para la co-creación o evaluación del producto.

- Enfoque del ecosistema: en el desarrollo y evaluación del producto o servicio, se debe contar con la interacción de todos los usuarios e interesados (empresas, investigadores y usuarios finales).

- Nivel de apertura: el proceso de innovación debe ser lo más abierto posible, contando con la participación del mayor número de usuarios posibles. En relación con esta condición se debe considerar también el grado de intercambio de conocimientos y el grado de aceptación de nuevos socios o usuarios.

- Comunidad: los usuarios participantes de estos entornos deben formar parte de una comunidad de interés o de práctica que puede estar o no geográficamente limitada, pero que comparte características y/o intereses.

- Vida útil: los proyectos desarrollados en estos entornos existen durante un período de tiempo acotado, aunque el Living Lab tenga vocación de continuidad.

- Escala: hace referencia al número de usuarios involucrados en las distintas actividades de investigación e innovación desarrolladas dentro del Living Lab. El mismo se debe definir a nivel global del Living Lab, ya que el tipo de innovación abordada en cada proyecto definirá cuántos usuarios pueden participar en estos.

- Contexto real: los usuarios deben ser estudiados dentro de un contexto real, que reproduzca el entorno natural de los usuarios tanto como sea posible. 
En segundo lugar, el entorno de los Living Labs se define por sus elementos metodológicos y de desarrollo ${ }^{4}$, a saber:

- Evaluación, investigación y co-creación: dentro del entorno del Living Lab los usuarios participan en diferentes fases del ciclo de innovación de un producto o servicio. En este proceso es fundamental que interactúen con los investigadores y que su participación sea evaluada mediante técnicas como las encuestas o entrevistas en profundidad.

- Rol del usuario: en los Living Labs los usuarios pueden ser informantes, evaluadores, colaboradores y co-creadores. En todo caso, sus roles dependerán de la visión y objetivo que se persiga y del grado de acción de estos dentro de las actividades.

El tercer y último componente de los Living Labs son sus resultados de innovación. El conocimiento de los resultados tangibles permitirá evaluar el impacto y determinar qué enfoques funcionan mejor, permitiendo mejorar la implementación de futuros proyectos vinculados al Living Lab .

A pesar de los avances en la conceptualización de los Living Labs como los apuntados en párrafos precedentes, la investigación realizada en torno a los mismos es reciente, se remonta a poco más de una década ${ }^{2,5}$, lo que explica su desconocimiento por parte de la comunidad profesional relacionada con la intervención social y educativa. Algunos autores, como Veeckman et al. ${ }^{4}$ apuntan que este incipiente campo de estudio se ha enfrentado con dificultades diversas que han limitado el avance de la investigación. En la misma línea, Schuurman et al. ${ }^{14}$, tras realizar una revisión sistemática de literatura en torno a los Living Labs, señalan los escasos avances tanto teóricos como empíricos en este campo, lo que atribuyen en parte a la indefinición del término, traducido en una falta homogeneidad de formatos de Living Lab. A todo ello habría que sumar también la diversidad de diseños, objetivos o potenciales usuarios objetivos de los mismos, etc. ${ }^{15}$.

Por este motivo, en este trabajo planteamos conocer el estado de la literatura producida desde las ciencias sociales, identificando y analizando resultados de estudios que aporten información de interés sobre este tipo de laboratorios, que ayuden a una mejor delimitación y avance del conocimiento en este campo. En particular, nos interesa aquí especialmente lo que la literatura tiene por decir sobre un tipo específico de Living Lab que tienen su foco puesto en las personas mayores, los denominados Senior Living Labs. De esta manera, esperamos conocer lo que esta propuesta de innovación abierta tiene para ofrecer a este grupo de edad, así como a las actuales sociedades envejecidas. Para ello proponemos una Revisión Sistemática de la Literatura que responda a las siguientes preguntas de investigación: a) ¿cuál es el contexto geográfico en el que se ubican y a qué tipo de usuarios se dirigen los Living Senior Labs?, b) ¿cuáles son sus objetivos?, c) ¿qué tipo de estudios se realizan en los Living Senior Lab? y d) ¿cuáles son las características que comparten? 


\section{Metodología}

Para tratar de dar respuesta a estas cuestiones se realizó una revisión sistemática de literatura (RSL) sobre Living Senior Labs en ciencias sociales. La metodología empleada se caracterizó por el desarrollo sistemático y explícito de las siguientes fases: planteamiento de preguntas de investigación, establecimiento de criterios de selección, desarrollo de estrategias de búsqueda, selección de estudios, análisis y extracción de la información y comunicación de los resultados ${ }^{16,17}$.

A partir de las preguntas señaladas, los criterios de selección para el refinamiento de la muestra se resumen en la Figura 2. Con respecto a las estrategias de búsqueda de literatura, esta investigación se limitó a las bases de datos que presentan un mayor impacto científico en el área de las ciencias sociales: Web Of Science (WOS) y Scopus. Para acceder al se establecieron los siguientes algoritmos de búsqueda: "living $A N D$ lab AND technolog*", "living AND lab AND gerontechnolog*", "living AND lab AND senior*", "living AND lab AND elder*", "living AND lab AND age"," "living AND lab AND old $A N D$ adult", "living AND lab AND old* AND people", "senior* AND lab", "senior* AND lab AND age*". Estos algoritmos se aplicaron al título del recurso empleando los filtros correspondientes.

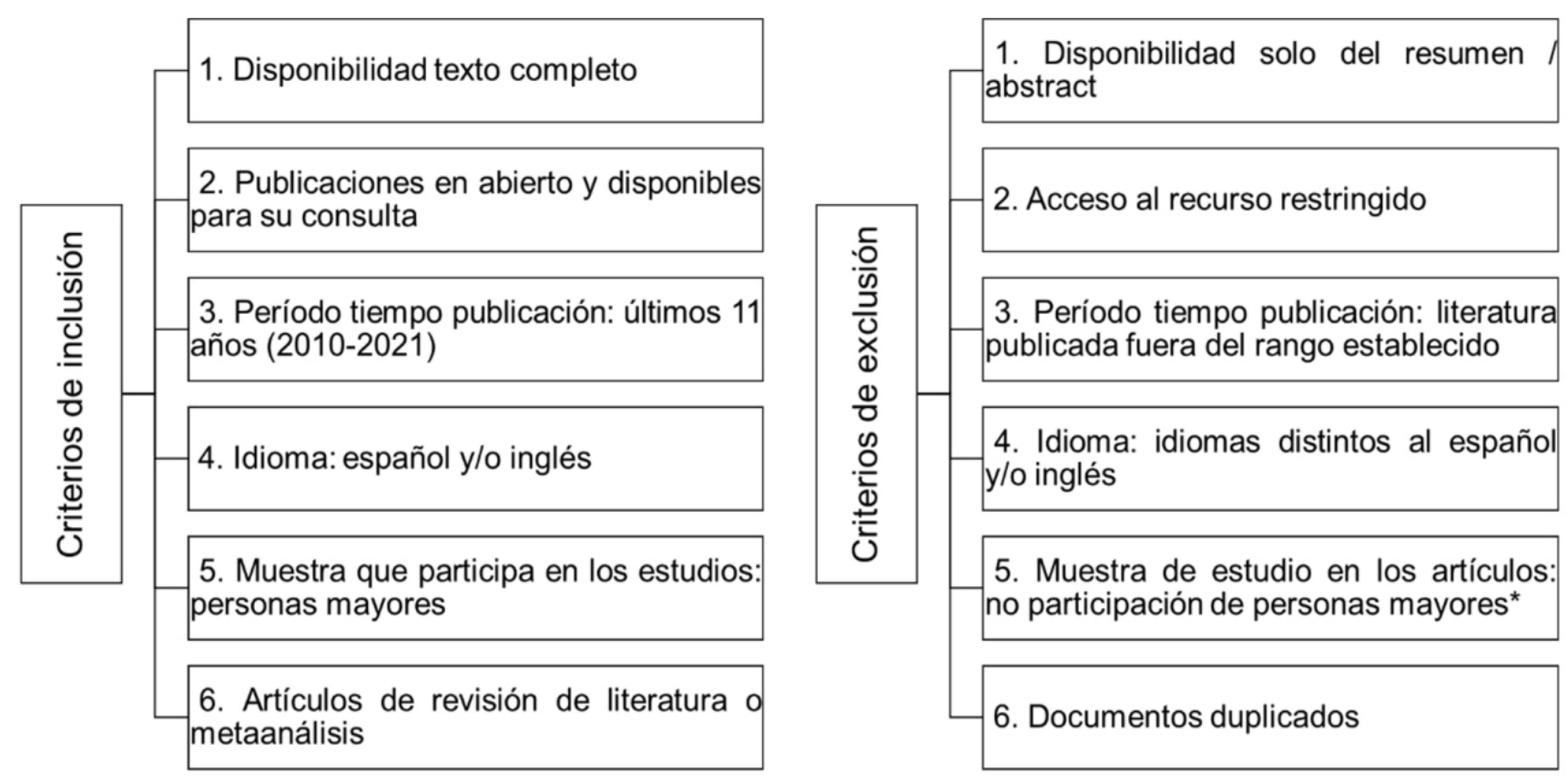

*Nota: este criterio no se aplicó en aquellos artículos en los que se realizaba una revisión de literatura o metaanálisis.

Figura 2. Criterios de inclusión y exclusión.

Fuente: Elaboración propia. 
Como se indica en la Figura 3, una primera búsqueda arrojó 4.374 entradas en WOS y 135.948 en Scopus. Para la selección final de los artículos que componen la muestra, se realizó un proceso de dos fases. La primera constó de la aplicación de los criterios de inclusión y exclusión establecidos. Y la segunda, se basó en una lectura exploratoria y de calidad, valorando la pertinencia y relevancia del contenido presentado en los diferentes artículos evaluando la idoneidad de estos para dar respuesta a las preguntas de investigación planteadas. Al finalizar estos procesos, 19 artículos se convirtieron en el material de análisis para este trabajo.

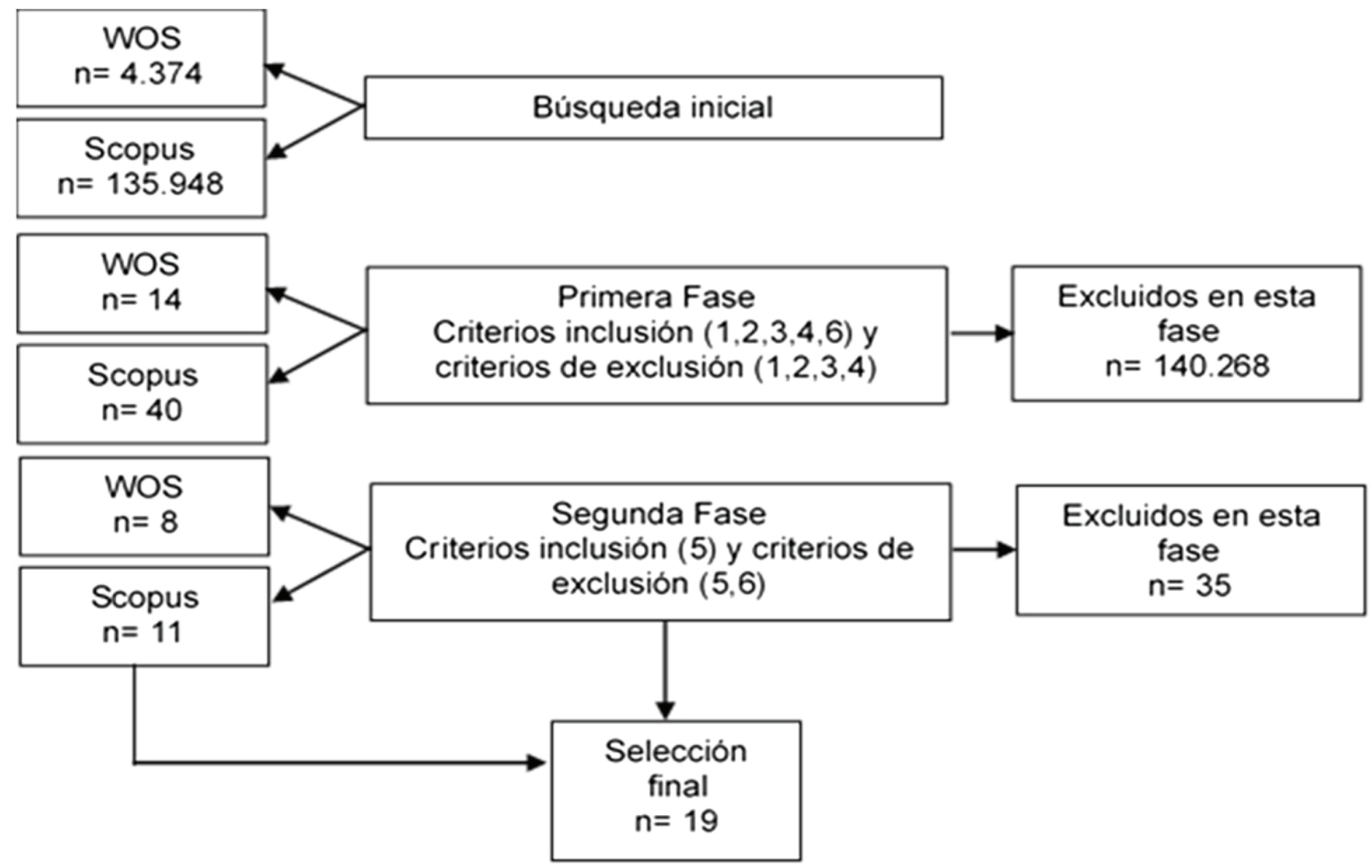

Figura 3. Proceso de selección de la literatura.

Fuente: Elaboración propia.

A partir de aquí, se llevó a cabo una lectura en profundidad de los artículos finalmente seleccionados. De cada uno de estos se extrajo la siguiente información: autor/es, año de publicación, título del artículo, tipo de estudio realizado, objetivos presentados en el artículo, contexto geográfico, usuarios o participantes y características del Living Senior Lab. Los resultados obtenidos se presentan a continuación. 


\section{Resultados}

\section{Caracterización del material analizado}

En la Tabla 1 aparecen, de manera resumida, los principales datos sobre el material sometido a análisis. Como puede apreciarse, la mayoría de estas investigaciones se han llevado a cabo utilizando metodología de investigación cualitativa. De los 19 documentos, 14 optan por este enfoque metodológico, en su mayor parte utilizando como técnica de recolección de datos la entrevista. En menor medida, se recurre al uso de otras herramientas de investigación como los grupos focales ${ }^{13} \mathrm{o}$ estudios de casos $^{18}$ para complementar la información obtenida. También se encuentran estudios cualitativos basados en revisión de literatura, por ejemplo, los trabajos desarrollados por Angelini et al. ${ }^{19}$, Verloo et al. ${ }^{7}$, Zerwas y Von Kortzfleish ${ }^{20}$. Por otro lado, algunas investigaciones se centran en la descripción de experiencias desarrolladas en el marco del laboratorio ${ }^{21-27}$. Cabe reseñar que cinco de los estudios emplearon una metodología mixta, conjugando el uso de entrevistas semiestructuradas y cuestionarios para la evaluación y valoración de diversos tipos de recursos ${ }^{28-32}$.

En relación con el lugar de realización de los estudios y la ubicación de los propios Senior Labs, los estudios se focalizan en su mayor parte en el contexto europeo. La literatura reporta casos de distintos países como Alemania, España, Grecia, Francia, Italia, Países Bajos, Suiza y Reino Unido. Tan solo uno de los trabajos analizados se realizó en el contexto asiático ${ }^{23}$.

Por último, en la mayor parte de las investigaciones analizadas se indica que en el proceso de recolección de datos participaron exclusivamente los usuarios mayores (por ejemplo, Alaoui \& Lewkowicz ${ }^{28}$; Vaziri et al. ${ }^{29}$; Wu et al. ${ }^{30}$; Konstantinidis et al. ${ }^{31}$. En algunos estudios se amplía la recogida de información también a familiares de las personas mayores, a investigadores del laboratorio ${ }^{13,32-34} \mathrm{o}$ a diferentes profesionales vinculados al Living $\operatorname{Lab}^{18}$. 
Tabla 1. Metodología de los estudios, contexto geográfico y usuarios principales de los Living Senior Labs

\begin{tabular}{|c|c|c|c|c|}
\hline Autor/es artículo & Año publicación & Tipo de estudio realizado & $\begin{array}{l}\text { Contexto geográfico del } \\
\text { Living Lab }\end{array}$ & Usuario/s del Living Lab \\
\hline Zerwas y Von Kortzfleish & 2011 & Cualitativo (revisión de literatura) & Europa (Distintos países) & Mayores \\
\hline Kang & 2012 & $\begin{array}{l}\text { Cualitativo (descripción de } \\
\text { experiencia) }\end{array}$ & Asia (Taiwán) & Mayores \\
\hline Wu et al. & 2014 & $\begin{array}{l}\text { Mixto (cuestionarios } \\
\text { estandarizados, entrevistas } \\
\text { semiestructuradas y grupos } \\
\text { focales) }\end{array}$ & Europa (Francia) & Mayores \\
\hline Blain et al. & 2014 & $\begin{array}{l}\text { Cualitativo (descripción de } \\
\text { experiencia) }\end{array}$ & Europa (Francia) & Mayores \\
\hline Alaoui y Lewkowicz & 2015 & $\begin{array}{l}\text { Mixto (cuestionarios y } \\
\text { entrevistas) }\end{array}$ & Europa (Francia) & Mayores \\
\hline Konstantinidis et al. & 2016 & $\begin{array}{l}\text { Mixto (evaluación de recursos y } \\
\text { entrevistas) }\end{array}$ & Europa (Grecia) & Mayores \\
\hline Riva-Mossman et al. & 2016 & $\begin{array}{l}\text { Cualitativo (descripción de } \\
\text { experiencia) }\end{array}$ & Europa (Suiza) & Mayores \\
\hline Vaziri et al. & 2016 & $\begin{array}{l}\text { Mixto (cuestionarios y } \\
\text { entrevistas) }\end{array}$ & $\begin{array}{l}\text { Europa (Alemania y España) } \\
\text { y Australia (Sydney) }\end{array}$ & Mayores \\
\hline Angelini et al. & 2016 & Cualitativo (revisión de literatura) & Europa (Distintos países) & $\begin{array}{l}\text { Mayores, familias y equipo } \\
\text { sanitario }\end{array}$ \\
\hline Calyam et al. & 2017 & $\begin{array}{l}\text { Cualitativo (descripción de } \\
\text { experiencia) }\end{array}$ & Estados Unidos & Mayores \\
\hline Noublanche et al. & 2019 & $\begin{array}{l}\text { Cualitativo (descripción de } \\
\text { experiencia) }\end{array}$ & Europa (Francia) & Mayores \\
\hline Van Den Kieboom et al. & 2019 & $\begin{array}{l}\text { Mixto (entrevistas, cuestionarios } \\
\text { y grupos focales) }\end{array}$ & Europa (Países Bajos) & $\begin{array}{l}\text { Mayores y cuidadores } \\
\text { informales }\end{array}$ \\
\hline Callari et al. & 2019 & $\begin{array}{l}\text { Cualitativo (entrevistas } \\
\text { semiestructuradas) }\end{array}$ & Europa (Reino Unido) & Mayores \\
\hline Callari et al. & 2020 & $\begin{array}{l}\text { Cualitativo (entrevistas } \\
\text { semiestructuradas) }\end{array}$ & Europa (Reino Unido) & Mayores \\
\hline Marone et al. & 2020 & $\begin{array}{l}\text { Cualitativo (entrevistas } \\
\text { semiestructuradas y estudios } \\
\text { de casos) }\end{array}$ & Europa (Italia) & Mayores y profesionales \\
\hline Noublanche et al. & 2020 & $\begin{array}{l}\text { Cualitativo (descripción de } \\
\text { experiencia) }\end{array}$ & Europa (Francia) & Mayores \\
\hline Verbeek et al. & 2020 & $\begin{array}{l}\text { Cualitativo (descripción de } \\
\text { experiencia) }\end{array}$ & Europa (Países Bajos) & Mayores y familias \\
\hline Verloo et al. & 2020 & Cualitativo (revisión de literatura) & Europa (Suiza) & Mayores \\
\hline Rumeau et al. & 2021 & $\begin{array}{l}\text { Cualitativo (entrevistas y grupos } \\
\text { focales) }\end{array}$ & Europa (Francia) & Mayores \\
\hline
\end{tabular}




\section{Tipo de actividades y principales productos desarrollados en los Senior Labs}

Los objetivos que buscan los Senior Labs son enunciados habitualmente en términos de la mejora de la calidad de vida de las personas mayores. Estos objetivos están orientados a atender al cuidado físico y el bienestar integral, favorecer la autonomía e independencia en la realización de actividades de vida diaria, mejorar la calidad funcional a través de diferentes servicios de cuidado y apoyo, y atender el desarrollo e interacción social de los mayores. En relación con estos objetivos pueden identificarse una serie de productos y actividades que son descritos en los documentos analizados y que son parte y resultado del funcionamiento de los Senior Labs (ver, Tabla 2).

\section{Productos para el cuidado integral y salud física}

Un ejemplo de este tipo de producto aparece en el trabajo de Calyam et al. ${ }^{22}$ en la descripción del sistema "ElderCare-as-a-SmartService" (ECaaS), que integra aplicaciones orientadas al monitoreo de la salud de los mayores en sus hogares, así como al entrenamiento de fisioterapia de forma remota. Se trata de un entorno de innovación virtual basado en la nube que recrea un entorno de vida real para el cuidado de las personas mayores. En el trabajo de Konstantinidis et al. ${ }^{31}$ se presenta también la descripción de desarrollos, como la plataforma exergaming web de cuerpo completo, denominada webFitForAll (wFFA). Esta plataforma se diseñó con el propósito de fomentar el ejercicio físico de las personas mayores y el mantenimiento y avance del estado físico saludable y el bienestar físico de los usuarios. Se trata de valorar esta herramienta de cara a aumentar su facilidad de uso, y su grado de aceptación por parte de usuarios finales de edades avanzadas.

\section{Productos para la salud mental y las enfermedades del sistema nervioso}

Kang ${ }^{23}$, describe el Living Lab de Suan-Lien, en el Center of Innovation and Synergy for Intelligent Home and Living Technology (INSIGHT) de la Universidad de Taiwán. En este laboratorio se sigue una línea de trabajo en torno a los juegos serios. Un ejemplo de ello es el "TaoGei”, que permite a los mayores utilizar dispositivos móviles para el entrenamiento de la memoria y apoyo de terapias de reminiscencia. Otro producto desarrollado en este Senior Lab es el denominado "Tempo.Tempo", dispositivo interactivo que mezcla estímulos visuales y auditivos y que está destinado a la realización de ejercicios de rehabilitación para personas con Parkinson.

Otro ejemplo de este tipo de productos, relacionado con la demencia, es el Living Lab vinculado al Data Drive Research and Innovation Programme (DDRI) de la Universidad de Coventry (Reino Unido), descrito en Callari et al. ${ }^{33,34}$. El desarrollo de este ecosistema de innovación se lleva a cabo en dos tipos de entornos. El "Entorno A" ofrece cuidados residenciales diurnos a largo plazo, así como cuidados de relevo a corto plazo para mayores con demencia. Y el "Entorno B" ofrece un espacio de vida independiente para mayores de 55 años, en el que se presta apoyo y atención para quienes lo necesiten. En ambos entornos se puede identificar diferentes niveles de participación de los usuarios, desde el desempeño de una actividad de co-creación hasta la participación en la prueba y 
evaluación de productos y/o servicios del Living Lab. En una línea similar, Marone et al. ${ }^{18}$ presentan el análisis y estudio del Lecco Innovation Living Lab (LILL) ubicado al norte de Italia. Mediante el trabajo realizado en este entorno, se busca identificar las necesidades de los usuarios con la finalidad de mantener la participación de estos en la co-creación de tecnologías para la neuro-rehabilitación.

Por último, Van Den Kieboom et al..$^{32}$ describen el desarrollo de un Living Senior Lab ubicado en la Provincia de Brabante Septentrional (Países Bajos) denominado Innovate Dementia 2.0. El objetivo de este entorno consiste en explorar las necesidades de personas mayores con demencia y de sus familiares. Tras la detección de dichas necesidades, ambos grupos (mayores y familias) participaron en el desarrollo, prueba y evaluación de dispositivos con el objetivo de satisfacer, de forma eficaz, las necesidades detectadas.

Tabla 2. Actividades y productos desarrollados en los Senior Labs

\begin{tabular}{|c|c|}
\hline $\begin{array}{l}\text { Autor/es (año } \\
\text { publicación } \\
\text { artículo) }\end{array}$ & Actividad desarrollada por los usuarios mayores \\
\hline $\begin{array}{l}\text { Alaoui y } \\
\text { Lewkowicz } \\
(2015)\end{array}$ & $\begin{array}{l}\text { Participación en el entorno del Living Lab: enriquecimiento y } \\
\text { perfeccionamiento del producto (maquetas y prototipos) dentro de un } \\
\text { entorno controlado para valorar su usabilidad. Participación en el entorno } \\
\text { hogar familiar: evaluación y prueba del producto en condiciones de vida } \\
\text { real. En un primer momento, se realizaron sesiones individuales con los } \\
\text { participantes, y, posteriormente, las pruebas se desarrollados entre los } \\
\text { participantes en parejas para que realizaran tareas de interacción. Para } \\
\text { recoger la información emitida por parte de los mayores se realizaron } \\
\text { entrevistas y cuestionarios de evaluación de los productos. }\end{array}$ \\
\hline
\end{tabular}

Tipo de producto/servicio Participación de los mayores en pruebas de usabilidad de los productos

Kang et al. (2012)

\section{realizada en entornos reales.}

Tras finalizar la evaluación de productos, se realizaron entrevistas para informar y recoger opiniones de los participantes con el objetivo de mejorar los productos y su usabilidad.
Aplicación: juego serio de memoria (TaoGei). Dispositivo interactivo: para terapia con enfermos de Parkinson (Tempo Tempo).
Dispositivo interactivo: televisión interactiva "segunda pantalla" que facilitan la interacción remota entre las personas mayores y sus familiares
Evaluación de exergaming dentro de un entorno de laboratorio vivo. Para la prueba del prototipo, los participantes realizaron las sesiones de ejercicio utilizando una televisión inteligente, tabletas y móviles Konstantinidis et al. (2016) evaluar la funcionalidad del sistema, los mayores participantes fueron entrevistados y valoraron aspectos como la satisfacción, dificultad de uso, utilidad hacia el envejecimiento activo. inteligentes, probando diferentes opciones incluidas en el producto. Para

Exergaming: webFitForAll (wFFA)

Participación en el proceso de co-diseño y co-desarrollo de tecnologías y Noublanche et productos gerontecnológicos para mejorar la atención hospitalaria. Esta al. $(2019,2020)$ participación se planteó en dos entornos: talleres incubadora de ideas y Tipo de producto/servicio sin especificar habitación de hospital experimental.
La participación de los mayores se produjo en dos fases. En primer lugar, se realizaron entrevistas individuales y un grupo focal basado en los resultados obtenidos en las entrevistas previas para detectar Rumeau et al. en los resultados obtenidos en las entrevistas previas para detectar
(2021) los participantes experimentaron, en un entorno Living Lab, las 


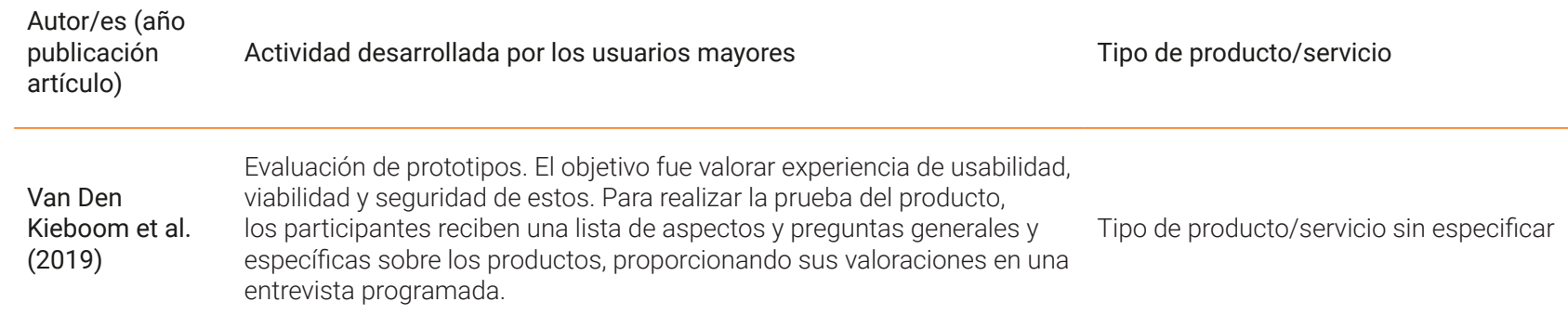

Evaluación de un robot interactivo dentro de un entorno Living Lab. La participación de los mayores se produjo durante 4 sesiones. En la primera se presentó el producto y sus principales funciones. Durante las Wu et al. (2014) sesiones restantes los participantes realizaron una serie de tareas para simular el uso del robot en un contexto real y cotidiano, aumentando progresivamente la dificultad de estas. Para registrar las valoraciones de los participantes, se aplicó un cuestionario de aceptación del robot y, de forma complementaría, se realizó una entrevista.

\section{Productos para favorecer la autonomía e independencia de los mayores}

Noublanche et al..$^{24,25}$, presentan el Living Lab denominado Angers Living Lab En Gériatrie Hospitalière (ALLEGRO), vinculado al Departamento de Geriatría del Hospital Universitario de Angers (Francia). Los objetivos de este Senior Living Lab se centran en mejorar el entorno vital de las personas mayores, compensar su pérdida de independencia y permitirles vivir en sus hogares el mayor tiempo posible. Para conseguir dichos objetivos, el laboratorio cuenta con dos tipos de servicios. El primero se basa en "talleres incubadores de ideas", en los que se reúnen y participan tanto los desarrolladores de gerontecnología como los usuarios finales del hospital. Y, el segundo, es el denominado "habitación de hospital experimental” destinado a probar la eficacia diagnóstica de los prototipos diseñados.

En esta línea, Wu et al ${ }^{30}$ presentan el proceso de evaluación y aceptación de robots por parte de personas mayores, con la finalidad de mantener un estilo de vida independiente y mejorar su autonomía. Esta evaluación se llevó a cabo en el LUSAGE Gerontechnology Living Lab (Francia), en este entorno los usuarios finales interactuaron, una vez a la semana y durante un mes, con un robot llamado "Kompaï”. Los resultados generales de este estudio se orientan hacia una aceptación generalizada de este dispositivo por parte de personas mayores de edades no muy avanzadas.

También en Francia, Rumeau et al. ${ }^{13}$ describe otra experiencia en la que se buscaba dar respuesta al problema de personas mayores que vivían en contextos rurales, proponiendo entornos de viviendas compartidas. El objetivo de este proyecto se concretó en identificar herramientas y soluciones tecnológicas que pudieran mejorar el servicio brindado a los residentes de dichas viviendas, optimizando así las actividades diarias de estos. Para la consecución de este objetivo, las personas mayores realizaron diferentes pruebas de las herramientas tecnológicas presentes en una casa inteligente llamada Blagnac, siendo esta una plataforma tecnológica que se está utilizando para el diseño y la evaluación de tecnologías de apoyo a una mayor autonomía de salud y en el hogar. 


\section{Productos para mejorar la calidad funcional de los mayores}

Verbeek et al. ${ }^{27}$ realizan un análisis del Living Lab in Ageing and Long-Term Care fundado en los Países Bajos. En este estudio, los autores describen el Living Lab como una red en la que los investigadores colaboran tanto con las personas mayores como con sus familias, distintos profesionales, directores de asistencia sanitaria, responsables políticos y profesores. Todo esto con el objetivo de mejorar no solo la calidad de vida de los mayores y sus familias, sino también la calidad funcional mediante la prestación de servicios de cuidados de corta y larga duración a domicilio y en entornos institucionales de carácter paliativo y de rehabilitación geriátrica. Por su parte, Blain et al. ${ }^{21}$, describen la experiencia del Senior Living Lab denominado Falls-MACVIAR-LR, ubicado en Languedoc-Rosellón (Francia). Este entorno desarrolla un trabajo transversal desde la Asociación Europea de Innovación sobre el Envejecimiento Activo y Saludable, estableciendo como su principal objetivo el desarrollo de productos (dispositivos) y servicios para la prevención de caídas de las personas mayores. De manera similar, Vaziri et al. ${ }^{29}$ llevaron a cabo un estudio sobre el proceso de co-creación de un tipo de juego (exergaming) denominado iStoppFalls, dirigido a la prevención de caídas en personas mayores. El objetivo aquí fue identificar los factores que influyen en la usabilidad, la experiencia del usuario y la aceptación de estos juegos por parte de los mayores.

\section{Productos para favorecer el desarrollo e interacción social de los mayores}

Alaoui y Lewkoicz ${ }^{28}$ presentan un Living Lab orientado a fines sociales. El mismo fue desarrollado dentro de un proyecto europeo de televisión social denominado FoSiBLE, con base en Francia. Su objetivo era promover las interacciones sociales de los mayores para aumentar su bienestar y autoestima a través del uso de dispositivos de comunicación e intercambio de información. El diseño de los dispositivos se llevó a cabo en dos fases. En la primera se realizó una prueba del sistema en un entorno de laboratorio controlado durante varias sesiones, con la participación de usuarios mayores y diferentes profesionales. En la segunda fase, se realizaron pruebas en los hogares de los participantes en condiciones de vida real. Durante ambas fases se buscó garantizar que el funcionamiento y el uso del dispositivo se adaptara a las expectativas del usuario, ofreciéndole una experiencia adecuada.

Otro ejemplo de estudios en los que se presenta una perspectiva social de los Living Labs, es el de la Universidad de Artes y Ciencias Aplicadas de Suiza Occidental. En un proyecto de investigación interdisciplinario e interprofesional realizado por Riva-Mossman et al. ${ }^{26}$ se describe el diseño de una plataforma para la reinvención del concepto de envejecimiento mediante el cambio de actitudes sociales y la participación de los mayores. Para ello se plantearon metodologías con enfoques de construcción social, la investigación-acción, investigación narrativa, entre otras ${ }^{26}$. 


\section{Características de los Senior Labs}

A pesar de la variedad de proyectos y productos generados por los Senior Labs, el análisis de los artículos seleccionados ha permitido identificar una serie de elementos que podrían caracterizarlos genéricamente. En primer lugar, la mayor parte de los estudios entienden estos entornos como espacios de innovación abierta destinados a la construcción e intercambio de conocimientos, que permitan la co-creación de productos y o el diseño de servicios adaptados a situaciones de vida real de los mayores $13,18,20,22-26,28,30,34$. Asimismo, existe un amplio acuerdo en que los Living Senior Labs deben dotarse de una infraestructura que favorezca la participación de los usuarios durante todo el proceso de diseño y prueba de los nuevos productos o prototipos ${ }^{13,28-32}$.

El nivel y tipo de participación de los mayores puede variar de unos Senior Labs a otros. Lo común es que los mayores asuman un rol de informantes, mediante la implementación de cuestionarios, participación en grupos focales o entrevistas. Sin embargo, también es común que se busque su implicación directa en ensayos, pruebas y/o experimentación de productos ${ }^{7,13,18-20,23-26,28,30,33,34}$. La implicación activa de los mayores en todas las fases de la innovación tecnológica fue, sin embargo, minoritaria. Lo anterior es llamativo, teniendo en cuenta que en la literatura se suele enfatizar esta cuestión como deseable.

Otro rasgo común y característico de los Senior Labs es que posibilitan y promueven la interacción entre distintos actores participantes en estos entornos, estableciendo redes de colaboración entre usuarios, investigadores, productores, y otros actores externos $^{18-20,23-28,30,33,34}$. Por esta vía, se espera que la participación y el establecimiento de redes de innovación no solo beneficie a las personas mayores y a sus familias, sino también a los equipos de investigación y desarrollo, aumentando así la posibilidad de que sus propuestas sean aceptadas y adoptadas por un mayor número de usuarios finales ${ }^{13,18,19,22-25,30}$. Más aún, la idea es que los beneficios estimados de la actividad desarrollada en los Senior Labs se extienda también a distintos sectores de la sociedad. En este sentido, se mencionan con frecuencia el ámbito sanitario y de cuidado asistencial (telemedicina y teleasistencia), o el de la industria, en particular la interesada en productos relacionados con la domótica, $718,19,21,22,24-27,29,32,33$.

\section{A modo de conclusión}

Nos planteamos en este trabajo recopilar y analizar la información más significativa sobre un tipo particular de Living Lab relacionado con procesos de envejecimiento. Para ello hemos revisado la literatura disponible publicada en las principales bases de datos científicas en el campo de las ciencias sociales a través de la metodología de la Revisión Sistemática de Literatura. En el análisis y la presentación de los resultados hemos considerado la propuesta de Veeckman et al. ${ }^{4}$ sobre elementos básicos de los Living Labs (enfoque, entorno, y resultados de la innovación). 
A partir de la revisión del material publicado, encontramos que los estudios sobre este tema han sido mayoritariamente cualitativos, han supuesto la participación directa de personas mayores, y han estado enfocados en el examen de Living Labs europeos. Estos laboratorios vivos han orientado sus actividades hacia el horizonte de una mayor calidad de vida para las personas de edad avanzada. Lo han hecho a través de la co-creación y puesta a prueba de productos para el cuidado integral, el cuidado de la salud física y mental, el aseguramiento de la autonomía, la mejora de la calidad funcional de los mayores y e favorecimiento de su vida social. De este modo, el desarrollo de Living Senior Labs contribuye al objetivo de ir más allá de la mera alfabetización digital de este grupo de edad, buscando una implicación más directa en el proceso de creación y evaluación de productos y servicios con los que se espera cubrir sus necesidades

A pesar de la variedad de formatos o tipos de Living Senior Labs se han encontrado algunas características comunes a todos ellos. Estas características, podemos resumirlas en: a) presencia de entornos reales y de innovación abierta, b) contexto de intercambio de conocimientos y c) participación e interacción de los usuarios y otros interesados en la co-creación y evaluación de productos eficaces, útiles y adaptados a las necesidades de los usuarios (personas mayores).

\section{Limitaciones y futuras líneas de investigación}

Los resultados de la revisión sistemática han permitido identificar y delimitar determinados aspectos de los Living Senior Labs que pueden ayudar a su mejor conceptualización, conocimiento y expansión. Sin embargo, considerando el bajo número de artículos seleccionados, se destaca la necesidad de fortalecer esta línea de investigación a través de diversas vías. Por ejemplo, parece necesario complementar la Revisión Sistemática de la Literatura con otros métodos que ayuden a conocer a fondo la realidad de los Senior Labs. Esto puede incluir estudios de caso múltiple a nivel europeo, la aplicación de Análisis de Redes Sociales, o la ejecución de estudios etnográficos de casos concretos. Estos estudios han de explorar nuevas vías de análisis examinando los roles desempeñados por los usuarios mayores en este tipo de comunidades de innovación, explorando la orientación de los mismos en cuanto a su enfoque (técnico o social), o analizando la red de relaciones entre los diferentes laboratorios, y la tipología de entidades, instituciones o sectores empresariales que los promueven y apoyan. De alguna forma, estas investigaciones han de preocuparse por la forma como, alrededor de los retos de las poblaciones envejecidas, se establecen las relaciones entre ciencia, tecnología y sociedad. 


\section{Contribuciones de los autores}

Todos los autores participaron activamente en todas las etapas de la preparación del manuscrito.

\section{Financiamiento}

Esta investigación fue financiada por el Ministerio de Ciencia, Innovación y Universidades de España (Proyecto "Adaptabilidad subjetiva en entornos tecnosociales e innovación gerontecnológica basada en la vida, SENIORLAB-LBD, Ref. PID2019-107826GB-I00).

\section{Conflicto de interese}

Los autores no tienen ningún conflicto de interese que declarar.

\section{Derechos de autor}

Este artículo está bajo la Licencia Internacional Creative Commons 4.0, tipo BY (https://creativecommons.org/licenses/by/4.0/deed.es).

\section{(cc) BY}

\section{Editor}

Antonio Pithon Cyrino

Editora asociada

Katia Lerner

Presentado el

$16 / 06 / 21$

Aprobado el

09/08/21

\section{Referencias}

1. Bergvall-Kåreborn B, Ihlström Eriksson C, Ståhlbröst A. Places and Spaces within Living Labs. Technol Innov Manag Rev. 2015; 5(12):37-47. Doi: https://doi. org/10.22215/timreview951.

2. Leminen $S$, Rajahonka $M$, Westerlund $M$. Towards third-generation living lab networks in cities. Technol Innov Manag Rev. 2017; 7(11):21-35. Doi: https://doi. org/10.22215/timreview/1118.

3. Leminen $S$, Westerlund $M$. Towards innovation in Living Labs networks. Int J Prod Dev. 2012; 17(1-2):43-59. Doi: https://doi.org/10.1504/IJPD.2012.051161.

4. Veeckman C, Schuurman D, Leminen S, Westerlund M. Linking Living Lab characteristics and their outcomes: towards a conceptual framework. Technol Innov Manag Rev. 2013; 3(12):6-15. Doi: https://doi.org/10.22215/timreview748.

5. Bravo-Ibarra ER. Revisión sistemática del concepto de laboratorios vivos. Dimens Empres. 2020; 12(1):78-104. Doi: https://doi.org/10.15665/dem.v18i(1).2018.

6. European Network of Living Labs. 2019 Activity Report [Internet]. Brussels; 2019 [citado 25 Abr 2021]; 59. Disponible en: https://issuu.com/enoll/docs/activity_report_v6 
7. Verloo H, Lorette A, Gomes da Rocha C, Amoussou JR, Gillès de Pélichy E, Matos Queiros A, et al. A comprehensive scoping review protocol of using living labs to explore needs and solutions for older adults with dementia. Smart Homecare Technol Telehealth. 2020; 7:19-27. Doi: https://doi.org/10.2147/shtt.s233130.

8. Mitchell WJ. Me++: the cyborg self and the networked city. Cambridge: The MIT Press; 2004.

9. Ståhlbröst A. A living lab as a service: creating value for micro-enterprises through collaboration and innovation. Technol Innov Manag Rev. 2013; 3(11):37-42. Doi: https://doi.org/10.22215/timreview/744.

10. Irwin A. STS perspectives on scientific governance. In: Hackett EJ, Amsterdamska O, Lynch M, Wajcman J, editores. The handbook of science and technology studies. London: The MIT Press; 2008. p. 583-608.

11. Coorevits L, Jacobs A. Taking real-life seriously: an approach to decomposing context beyond "environment" in living labs. Technol Innov Manag Rev. 2017; 7(1):26-36. Doi: https://doi.org/10.22215/timreview/1047.

12. Følstad A. Living labs for innovation and development of information and communication technology: a literature review. Eletronic J Virtual Organ Netw. 2008; 10:99-131.

13. Rumeau P, Vigouroux N, Campo E, Bougeois E, Vella F, Van Den Bossche A, et al. Technological services in shared housing: needs elicitation method from home to living lab. IRBM. 2021; 42(2):73-82. Doi: https://doi.org/10.1016/j.irbm.2020.06.013.

14. Schuurman D, De Marez L, Ballon P. Living Labs: a systematic literature review. Open Living Lab Days 2015 [Internet]. 2015 [citado 29 Abr 2021]:1-17. Disponible en: https://biblio.ugent.be/publication/7026155/file/7026171.pdf

15. Burbridge M. If living labs are the answer - what's the question? A review of the literature. Procedia Eng. 2017; 180:1725-32. Doi: https://doi.org/10.1016/j. proeng.2017.04.335.

16. González Ferreira I, Urrútia G, Alonso-Coello P. Revisiones sistemáticas y metaanálisis: bases conceptuales e interpretación. Rev Esp Cardiol. 2011; 64(8):688-96. Doi: https:// doi.org/10.1016/j.recesp.2011.03.029.

17. Santana Martel JS, Pérez i Garcias A. El codiseño educativo haciendo uso de las TIC en educación superior: una revisión sistemática de literatura. EDUTEC Rev Electrónica Tecnol Educ. 2020; (74):25-50. Doi: https://doi.org/10.21556/edutec.2020.74.1799.

18. Marone L, Onofrio R, Masella C. The Italian case of lecco innovation living lab: stakeholders' needs and activities to contribute to the technological innovation process in healthcare. Sustainability. 2020; 12(4):1-15. Doi: https://doi.org/10.3390/su122410266.

19. Angelini L, Carrino S, Abou Khaled O, Riva-Mossman S, Mugellini E. Senior living lab: an ecological approach to foster social innovation in an ageing society. Future Internet. 2016; 8(4):1-19. Doi: https://doi.org/10.3390/fi8040050.

20. Zerwas D, Von Kortzfleisch HF. Potentials of living labs for the diffusion of information technology: a conceptual analysis. In: IFIP International Working Conference on Governance and Sustainability in Information Systems-Managing the Transfer and Diffusion of IT; 2011; Berlin. Berlin: Springer; 2011. p. 330-9.

21. Blain $\mathrm{H}$, Abecassis F, Adnet PA, Alomène B, Amouyal M, Bardy B, et al. Living lab falls-MACVIA-LR: the falls prevention initiative of the European innovation partnership on active and healthy ageing (EIP on AHA) in Languedoc-Roussillon. Eur Geriatr Med. 2014; 5(6):416-25. Doi: https://doi.org/10.1016/j.eurger.2014.07.010. 
22. Calyam P, Jahnke I, Mishra A, Antequera RB, Chemodanov D, Skubic M. Toward an ElderCare living lab for sensor-based health assessment and physical therapy. IEEE Cloud Comput. 2017; 4(3):30-9. Doi: https://doi.org/10.1109/MCC.2017.46.

23. Kang SC. Initiation of the Suan-Lien living lab - a living lab with an elderly welfare focus. Int J Autom Smart Technol. 2012; 2(3):189-99. Doi: https://doi.org/10.5875/ ausmt.v2i3.132.

24. Noublanche F, Jaglin-Grimonprez C, Sacco G, Lerolle N, Allain P, Annweiler C. The development of gerontechnology for hospitalized frail elderly people: the ALLEGRO hospital-based geriatric living lab. Maturitas. 2019; 125:17-9. Doi: https://doi. org/10.1016/j.maturitas.2019.04.002.

25. Noublanche F, Jaglin-Grimonprez C, Laignel L, Sacco G, Allain P, Annweiler C, et al. Adapting gerontechnological development to hospitalized frail older people: implementation of the ALLEGRO hospital-based geriatric living lab. J Am Med Dir Assoc. 2020; 21(4):550-4. Doi: https://doi.org/10.1016/j.jamda.2020.01.001.

26. Riva-Mossman S, Kampel T, Cohen C, Verloo H. The senior living lab: an example of nursing leadership. Clin Interv Aging. 2016; 11:255-63. Doi: https://doi.org/10.2147/ CIA.S97908.

27. Verbeek H, Zwakhalen SMG, Schols JMGA, Kempen GIJM, Hamers JPH. The living lab in ageing and long-term care: a sustainable model for translational research improving quality of life, quality of care and quality of work. J Nutr Health Aging. 2020; 24(1):43-7. Doi: https://doi.org/10.1007/s12603-019-1288-5.

28. Alaoui M, Lewkowicz M. Practical issues related to the implication of elderlies in the design process-The case of a Living Lab approach for designing and evaluating social TV services. IRBM. 2015; 36(5):259-65. Doi: https://doi.org/10.1016/j. irbm.2015.06.002.

29. Vaziri DD, Aal K, Ogonowski C, Von Rekowski T, Kroll M, Marston HR, et al. Exploring user experience and technology acceptance for a fall prevention system: results from a randomized clinical trial and a living lab. Eur Rev Aging Phys Act. 2016; 13(1):6. Doi: https://doi.org/10.1186/s11556-016-0165-z.

30. Wu Y-H, Wrobel J, Cornuet M, Kerherve H, Damnee S, Rigaud A-S. Acceptance of an assistive robot in older adults: a mixed-method study of human-robot interaction over a 1-month period in the Living Lab setting. Clin Interv Aging. 2014; 9:801-11. Doi: https://doi.org/10.2147/CIA.S56435.

31. Konstantinidis EI, Bamparopoulos G, Bamidis PD. Moving real exergaming engines on the web: the webFitForAll case study in an active and healthy ageing living lab environment. IEEE J Biomed Health Inform. 2016; 21(3):859-66. Doi: https://doi. org/10.1109/JBHI.2016.2559787.

32. Van Den Kieboom RC, Bongers I, Mark R, Snaphaan LJ. User-driven living lab for assistive technology to support people with dementia living at home: protocol for developing co-creation-based innovations. JMIR Res Protoc. 2019; 8(1):10952. Doi: https://doi.org/10.2196/10952.

33. Callari TC, Moody L, Saunders J, Ward G, Holliday N, Woodley J. Exploring participation needs and motivational requirements when engaging older adults in an emerging living lab. Technol Innov Manag Rev. 2019; 9(3):38-49. Doi: https://doi.org/10.22215/timreview/1223.

34. Callari TC, Moody L, Saunders J, Ward G, Woodley J. Stakeholder requirements for an ethical framework to sustain multiple research projects in an emerging living lab involving older adults. J Empir Res Hum Res Ethics. 2020; 15(3):111-27. Doi: https:// doi.org/10.1177/1556264619873790. 
Os Living Labs são experiências colaborativas que procuram envolver os cidadãos na governança científica e na avaliação tecnológica. Apesar de seu interesse, muito pouco se sabe sobre essas comunidades, seu funcionamento, tipologia e características. Portanto, foi realizada uma revisão sistemática da literatura sobre um tipo particular de Living Lab, orientado para os idosos: os Living Senior Labs. Com base em uma pesquisa geral nos principais bancos de dados científicos (WOS e Scopus) e na aplicação de critérios de inclusão pré-estabelecidos, foram selecionados 19 estudos sobre os Senior Labs (2010 a 2021). Os resultados proporcionam uma melhor compreensão deste tipo de ecossistema e criam uma base firme para o avanço do conhecimento neste campo.

Palavras-chave: Living labs. Senior labs. Idosos. Inovação aberta. Co-criação.

Living Labs are collaborative experiences that seek to involve citizens in scientific governance and technology assessment. In spite of their interest, very little is known about these communities, their functioning, typology and characteristics. Thus, a systematic literature review was carried out about a particular type of Living Lab, oriented towards the elderly: Living Senior Labs. Based on a general search in the main scientific databases (WOS and Scopus), and the application of pre-established inclusion criteria, 19 studies about Senior Labs were selected (2010 to 2021). The results provide a more comprehensive understanding of this type of ecosystem and create a strong foundation for progress in the knowledge of this area

Keywords: Living labs. Senior labs. Elderly. Open innovation. Co-creation. 